\title{
On Generalized Recurrent and Ricci Recurrent Lorentzian Trans-Sasakian Manifolds
}

\author{
V. J. Khairnar \\ N. V. P. Mandal's Arts, Commerce and Science College, Lasalgaon, Tal. Niphad, Dist. Nasik-422 306, M. S. \\ (INDIA)
}

\begin{abstract}
The purpose of the paper is to introduce the notion of generalized recurrent Lorentzian transSasakian manifold and study some of the properties of generalized recurrent and Ricci recurrent Lorentzian Trans-Sasakian manifolds.
\end{abstract}

Keywords: Local differential geometry, weakly symmetric, weakly Ricci symmetric, $\alpha$-Sasakian, $\beta$-Kenmotsu, Lorentzian Trans-Sasakian manifold.

\section{Introduction}

The notion of generalized recurrent manifolds was introduced by U. C. De and N. Guha [18]. A Riemannian manifold $\left(\mathrm{M}^{\mathrm{n}}, \mathrm{g}\right)(\mathrm{n}>2)$ is called generalized recurrent if its curvature tensor R satisfies the condition

$\left(\nabla_{\mathrm{X}} \mathrm{R}\right)(\mathrm{Y}, \mathrm{Z}) \mathrm{U}=\omega(\mathrm{X}) \mathrm{R}(\mathrm{Y}, \mathrm{Z}) \mathrm{U}+\mu(\mathrm{X}) \mathrm{f}$
where $\omega$ and $\mu$ are 1-forms with $\mu \neq 0$ and these are defined by

$$
\omega(X)=g(X, A), \quad \mu(X)=g(X, B)
$$

where $\mathrm{A}$ and $\mathrm{B}$ are the vector fields associated with 1 -forms $\omega$ and $\mu$ respectively.

In 1985, Oubina [7] introduced the notion of Trans-Sasakian manifolds which contains both the class of Sasakian \& cosympletic structures closed related to the locally conformal Kahler manifolds. Trans-Sasakian manifolds of type $(0,0),(\alpha, 0),(0, \beta)$ are the cosympleties, $\alpha$-Sasakian $\& \beta$-Kenmotsu manifold, respectively. In particular if $\alpha=1, \beta=0 \& \alpha=0, \beta=1$ then a Trans-Sasakian manifold reduces to a Sasakian and Kenmotsu manifold respectively. Thus Trans-Sasakian structures provide a large class of generalized quasi-Sasakian structures. In 2004 [11] studied on generalized recurrent Sasakian manifolds. In 2010 [17], studied on generalized $\phi$-recurrent trans-Sasakian manifolds. In 2002, Kim, Prasad \& Tripathi [9] studied generalized Ricci recurrent Trans-Sasakian manifolds. On the other hand, Many authors recently have studied Lorentzian $\alpha-$ Sasakian manifolds [2] and Lorentzian $\beta$ - Kenmotsu manifolds [4], [5]. In 2011, S.S.Pujar and V.J. Khairnar [14] have initiated the study of Lorentzian Trans-Sasakian manifolds and studied the basic results with some of its properties. Earlier to this, S. S. Pujar [16] has initiated the study of $\delta$-Lorentzian $\alpha$-Sasakian manifolds [10] and $\delta$ Lorentzian $\beta$-Kenmotsu manifolds [14]. Also, S. S. Pujar and V.J.Khairnar [15] have continued the work on Lorentzian manifolds and in fact studied the properties of weak symmetries of Lorentzian manifolds.

The object of the present paper is to study generalized recurrent and Ricci recurrent Lorentzian TransSasakian manifolds. Section 2 deals with preliminaries of Lorentzian Trans-Sasakian manifolds. However in section 3 we introduced the notion of generalized recurrent lorentzian trans-Sasakian manifold and obtain the relations between, the associated 1-forms $\omega$ and $\mu$ also obtained the separate theorem for the relationship between the 1-form $\omega$ and $\mu$. At the end of section 3, we generalize some of the results of [11] and some of those of [3]. Section 4 is devoted to a generalized Ricci recurrent Lorentzian Trans-Sasakian manifolds.

\section{Lorentzian Trans-Sasakian Manifolds}

Let $\mathrm{M}$ be lorentzian manifold of dimension 2n+1 [Prakasha, D. G. Bugewadi C. S. \& Basavavajappa N. S. 2008] with a lorentzian structure $(\phi, \xi, \eta, g)$ where $\phi$ is a $(1,1)$ tensor field, $\xi$ is a vector field, $\eta$ is a 1 -form and Lorentzian metric $\mathrm{g}$ which satisfy

$$
\begin{aligned}
& \eta(\xi)=-1, \varphi \xi=0, \eta(\varphi X)=0 \\
& \varphi^{2} \mathrm{X}=\mathrm{X}+\eta(\mathrm{X}) \xi, \mathrm{g}(\mathrm{X}, \xi)=\eta(\mathrm{X}) \\
& \mathrm{g}(\varphi \mathrm{X}, \varphi \mathrm{Y})=\mathrm{g}(\mathrm{X}, \mathrm{Y})+\eta(\mathrm{X}) \eta(\mathrm{Y}) \\
& \text { and } \mathrm{g}(\varphi \mathrm{X}, \mathrm{Y})=\mathrm{g}(\mathrm{X}, \varphi \mathrm{Y})
\end{aligned}
$$

for all $\mathrm{X}, \mathrm{Y}, \mathrm{C}^{\infty}$ vector fields on $\mathrm{M}$.

In Tanno.S.[12], classified the connected almost contact metric manifolds whose automorphism groups possesses the maximum dimension. For such a manifold the sectional curvature of the plane section containing $\xi$ 
is constant, say c. He showed that they can be divided into three classes. (1) Homogeneous normal contact Riemannian manifolds with $\mathrm{c}>0$. It is known that the manifolds of class (1) are characterized by admitting a Sasakian structure. Other two classes can be seen in Tanno [12].In Grey and Harvella [1], the classification of almost Hremitian manifolds, there appears a classW4 of Hermitian manifolds which are closely related to the conformal Kaehler manifolds. The class $\mathrm{C}_{6} \oplus \mathrm{C}_{5}[8]$ coincides with the class of the trans-Sasakian structure of type $(\alpha, \beta)$. In fact, the local nature of the two sub classes, namely $\mathrm{C}_{6}$ and $\mathrm{C}_{5}$ of trans-Sasakian structures are characterized completely. An almost contact metric structure on $\mathrm{M}$ is called a trans-Sasakian (please see details in [6] and [1]) if $(\mathrm{MxR}, \mathrm{J}, \mathrm{G})$ belongs to the class $\mathrm{W}_{4}$, where $\mathrm{J}$ is the almost complex structure on MxR defined by

$$
J\left(Z, f \frac{d}{d t}\right)=\left(\varnothing Z-f \xi, \eta(Z) \frac{d}{d t}\right)
$$

for all vector fields $\mathrm{X}$ on $\mathrm{M}$ and smooth function $\mathrm{f}$ on $\mathrm{MxR}$ and $\mathrm{G}$ is the product metric on MxR. This may be expressed by the condition

$$
(\nabla X \phi)(\mathrm{Y})=\alpha\{\mathrm{g}(\mathrm{X}, \mathrm{Y}) \xi-\eta(\mathrm{Y}) \mathrm{X}\}+\beta\{\mathrm{g}(\phi(\mathrm{X}), \mathrm{Y}) \xi-\eta(\mathrm{Y}) \phi(\mathrm{X})\},
$$

for any vector fields $\mathrm{X}$ and $\mathrm{Y}$ on $\mathrm{M}, \nabla$ denotes the Levi-Civita connection with respect to $\mathrm{g}, \alpha$ and $\beta$ are smooth functions on $\mathrm{M}$. The existence of condition (2.3) is ensured by the above discussion. In a Lorentzian Trans-Sasakian manifold $\mathrm{M}^{2 \mathrm{n}+1}(\phi, \xi, \eta, \mathrm{g})$ the following relation hold [13]

$$
\begin{aligned}
& \nabla_{X} \xi=-\alpha \varphi X-\beta\{X+\eta(X) \xi\} \\
& \left(\nabla_{\mathrm{X}} \eta\right)(\mathrm{Y})=\alpha \mathrm{g}(\varphi \mathrm{X}, \mathrm{Y})+\beta[\mathrm{g}(\mathrm{X}, \mathrm{Y})+\eta(\mathrm{X}) \eta(\mathrm{Y})] \\
& \mathrm{R}(\mathrm{X}, \mathrm{Y}) \xi=\left(\alpha^{2}+\beta^{2}\right)\{\eta(\mathrm{Y}) \mathrm{X}-\eta(\mathrm{X}) \mathrm{Y}\} \\
& +2 \alpha \beta\{\eta(\mathrm{Y}) \phi \mathrm{X}-\eta(\mathrm{X}) \phi \mathrm{Y}\} \\
& +(\mathrm{Y} \alpha) \varphi \mathrm{X}-(\mathrm{X} \alpha)(\varphi \mathrm{Y})+(\mathrm{Y} \beta) \varphi^{2} \mathrm{X}-(\mathrm{X} \beta) \varphi^{2} \mathrm{Y} \\
& \eta(\mathrm{R}(\mathrm{X}, \mathrm{Y}) \mathrm{Z})=\left(\alpha^{2}+\beta^{2}\right)[\eta(\mathrm{X}) \mathrm{g}(\mathrm{Y}, \mathrm{Z})-\eta(\mathrm{Y}) \mathrm{g}(\mathrm{X}, \mathrm{Z})] \\
& +2 \alpha \beta[\eta(X) g(\varphi \mathrm{Y}, \mathrm{Z})-\eta(\mathrm{Y}) \mathrm{g}(\varphi \mathrm{X}, \mathrm{Z})] \\
& -(Y \alpha) g(\varphi X, Z)+(X \alpha) g(\varphi Y, Z) \\
& -(Y \beta) g\left(\varphi^{2} X, Z\right)+(X \beta) g\left(\varphi^{2} Y, Z\right) \\
& \mathrm{S}(\mathrm{X}, \xi)=\left\{2 \mathrm{n}\left(\alpha^{2}+\beta^{2}\right)-\xi \beta\right\} \eta(\mathrm{X})+(2 \mathrm{n}-1)(\mathrm{X} \beta)-(\varphi \mathrm{X}) \alpha \\
& +(2 \alpha \beta \eta(X)+X \alpha) \Psi \\
& \mathrm{R}(\xi, \mathrm{Y}) \xi=\left\{\alpha^{2}+\beta^{2}-\xi \beta\right\} \varphi^{2} \mathrm{Y}+(2 \alpha \beta-\xi \alpha) \varphi \mathrm{Y} \\
& \mathrm{S}(\xi, \xi)=-2 \eta\left\{\alpha^{2}+\beta^{2}-\xi \beta\right\} \\
& 2 \alpha \beta-\xi \alpha=0
\end{aligned}
$$

For any vector field $\mathrm{X}, \mathrm{Y}, \mathrm{Z}$ on $\mathrm{M}$. Where $\mathrm{S}$ is Ricci curvature tensor of type $(1,1)$ and $\mathrm{Q}$ is Ricci operator given by

$$
\mathrm{S}(\mathrm{X}, \xi)=\mathrm{g}(\mathrm{QX}, \mathrm{Y}) \quad \text { and } \quad \psi=\mathrm{g}\left(\phi \mathrm{e}_{\mathrm{i}}, \mathrm{e}_{\mathrm{i}}\right)
$$

\section{Generalized Recurrent Lorentzian Trans-Sasakian Manifolds}

Definition3.1. A Lorentzian Trans-Sasakian manifold (M, g) of dimension $2 n+1$ is called generalized recurrent if its curvature tensor $\mathrm{R}$ satisfies the condition

$$
\left(\nabla_{\mathrm{x}} \mathrm{R}\right)(\mathrm{Y}, \mathrm{Z}) \mathrm{U}=\omega(\mathrm{X}) \mathrm{R}(\mathrm{Y}, \mathrm{Z}) \mathrm{U}+\mu(\mathrm{X})[\mathrm{g}(\mathrm{Z}, \mathrm{U}) \mathrm{Y}-\mathrm{g}(\mathrm{Y}, \mathrm{U}) \mathrm{Z}],
$$

Where $\omega$ and $\mu$ are 1-forms with $\mu \neq 0$ and these are defined by

$$
\omega(X)=g(X, A), \quad \mu(X)=g(X, B)
$$

Where $\mathrm{A}$ and $\mathrm{B}$ are the vector fields associated with 1 -forms $\omega$ and $\mu$ respectively.

\section{$\xi$-Sectional Curvature.}

The $\xi$-Sectional Curvature $\mathrm{K}(\xi, \mathrm{W})$ of a Lorentzian trans-Sasakian manifold for a unit vector field $\mathrm{W}$ orthogonal to $\xi$ is given by

From the relation (2.10) and (2.12) we have

$$
\mathrm{K}(\xi, \mathrm{W})=\mathrm{g}\{\mathrm{R}(\xi, \mathrm{W}) \xi, \mathrm{W}\}
$$

$$
\begin{aligned}
\mathrm{R}(\xi, \mathrm{W}) & \xi=\left\{\alpha^{2}+\beta^{2}-\xi \beta\right\}(\mathrm{W}+\eta(\mathrm{W}) \xi), \\
\mathrm{K}(\xi, \mathrm{W}) & =\mathrm{g}\{\mathrm{R}(\xi, \mathrm{W}) \xi, \mathrm{W}\} \\
& =\left\{\alpha^{2}+\beta^{2}-\xi \beta\right\}[\mathrm{g}(\mathrm{W}, \mathrm{W})+\eta(\mathrm{W}) \mathrm{g}(\xi, \mathrm{W})] \\
& =\left\{\alpha^{2}+\beta^{2}-\xi \beta\right\}
\end{aligned}
$$


Note that if the Lorentzian trans-Sasakian manifold $M$ is of non vanishing $\xi$-Sectional Curvature, then $\alpha^{2}+\beta^{2}-\xi \beta \neq 0$, and the Lorentzian trans-Sasakian manifold $M$ is of vanishing $\xi$-Sectional Curvature if and only if $\alpha^{2}+\beta^{2}-\xi \beta=0$.

Remark. For a Lorentzian $\beta$ - Kenmotsu manifold, $\xi$ - sectional curvature $K(\xi, X)=\beta^{2}-\xi \beta$.

For a Lorentzian $\alpha$-Sasakian manifold, $\xi$ - sectional curvature $\mathrm{K}(\xi, \mathrm{X})=\alpha^{2}$

For a Lorentzian Kenmotsu manifold, $\xi$ - sectional curvature $\mathrm{K}(\xi, \mathrm{X})=1$

For a Lorentzian Sasakian manifold, $\xi$ - sectional curvature $\mathrm{K}(\xi, \mathrm{X})=1$

Suppose a generalized recurrent weakly symmetric Lorentzian trans-Sasakian manifold is locally symmetric then $\nabla \mathrm{R}=0$ so that from (3.1) we have

Now (3.2) can be written as

$$
\omega(X) R(Y, Z) U+\mu(X)[g(Z, U) Y-g(Y, U) Z]=0
$$

Now contracting $\mathrm{Y}$ and $\mathrm{V}$ in (3.3), we get

$$
\begin{aligned}
& \omega(X) R(Y, Z, U, V)+\mu(X)[g(Z, U) g(Y, V)-g(Y, U) g(Z, V)]=0 \\
& \text { where } R(Y, Z, U, V)=g(R(Y, Z) U, V)
\end{aligned}
$$

Next put $Z=U=\xi$ in (3.4) we find

$$
\omega(X) S(Z, U)+2 n \mu(X) g(Z, U)=0
$$

For any vector field $X$ so that

$$
\left(\alpha^{2}+\beta^{2}-\xi \beta\right) \omega(X)-2 n \mu(X)=0
$$

$$
\left(\alpha^{2}+\beta^{2}-\xi \beta\right) \omega-2 n \mu=0
$$

Theorem3.1. If a generalized recurrent weakly symmetric Lorentzian trans-Sasakian manifold $M(n>1)$ of non vanishing $\xi$-sectional curvature is locally symmetric, then the1-form $\omega$ and $\mu$ are related by (3.5).

Corollary3.1. If a generalized recurrent weakly symmetric Lorentzian trans-Sasakian manifold $M(n>1)$ with non zero constant is locally symmetric, then the relation $\omega-2 \mathrm{n} \mu=0$ holds.

Theorem3.2. If a generalized recurrent weakly symmetric Lorentzian trans-Sasakian manifold M ( $\mathrm{n}>1$ ) of zero $\xi$-sectional curvature is locally symmetric, if and only if both 1-form $\omega$ and $\mu$ vanish.

Proof. For a locally symmetric space (3.2) holds. If the $\xi$ sectional curvature vanishes, then from (3.5), $\mu=0$. Again from (3.2), it follows that $\omega=0$. Second part is obvious from (3.1).

Corollary3.2. If a generalized recurrent weakly symmetric Lorentzian Sasakian manifold M ( $>1$ ) of zero $\xi$ sectional curvature is locally symmetric, if and only if both 1 -form $\omega$ and $\mu$ vanish.

Now taking $\mathrm{Y}=\mathrm{Z}=\mathrm{U}=\xi$ in (3.1) we get

$$
\left(\nabla_{\mathrm{x}} \mathrm{R}\right)(\xi, \xi) \xi=\omega(\mathrm{X}) \mathrm{R}(\xi, \xi) \xi+\mu(\mathrm{X})[\mathrm{g}(\xi, \xi) \xi-\mathrm{g}(\xi, \xi) \xi],
$$

By using (2.2), (2.10) in (3.6) we get

$$
\left(\nabla_{\mathrm{x}} \mathrm{R}\right)(\xi, \xi) \xi=0
$$

Now consider left hand side of (3.7),

$$
\left(\nabla_{X} R\right)(\xi, \xi) \xi=\nabla_{X}\{R(\xi, \xi) \xi\}-R\left(\nabla_{X} \xi, \xi\right) \xi-R\left(\xi, \nabla_{X} \xi\right) \xi-R(\xi, \xi) \nabla_{X} \xi
$$

Using the skew symmetric of the curvature tensor $\mathrm{R}$ the middle two terms in the above equation will vanish,

Also using the fact that $\mathrm{R}(\xi, \xi) \xi=0$ hence

Now by using (2.5), we get

$$
\begin{aligned}
\left(\nabla_{X} R\right)(\xi, \xi) \xi & =-R(\xi, \xi) \nabla_{X} \xi \\
& =R\left(\xi, \nabla_{X} \xi\right) \xi
\end{aligned}
$$

$$
\begin{aligned}
\left(\nabla_{\mathrm{X}} \mathrm{R}\right)(\xi, \xi) \xi= & \mathrm{R}\left(\xi, \nabla_{\mathrm{X}} \xi\right) \xi \\
= & \mathrm{R}(\xi,-\alpha \phi \mathrm{X}-\beta\{\mathrm{X}+\eta(\mathrm{X}) \xi\}) \xi \\
= & -\alpha \mathrm{R}(\xi, \phi \mathrm{X}) \xi-\beta \mathrm{R}(\xi, \mathrm{X}) \xi-\beta \eta(\mathrm{X}) \mathrm{R}(\xi, \xi) \xi \\
= & -\alpha\left\{\left(\alpha^{2}+\beta^{2}-\xi \beta\right) \phi \mathrm{X}+(2 \alpha \beta-\xi \alpha) \phi^{2} \mathrm{X}\right\} \\
& -\beta\left\{\left(\alpha^{2}+\beta^{2}-\xi \beta\right) \phi^{2} \mathrm{X}+(2 \alpha \beta-\xi \alpha) \phi \mathrm{X}\right\} \\
= & \left(\alpha^{2}+\beta^{2}-\xi \beta\right)\left[-\alpha \phi X-\beta \phi^{2} \mathrm{X}\right] \\
& +(2 \alpha \beta-\xi \alpha)\left[-\alpha \phi^{2} \mathrm{X}-\beta \phi \mathrm{X}\right]
\end{aligned}
$$

Now using (2.12), we get

$$
\left(\nabla_{X} R\right)(\xi, \xi) \xi=\left(\alpha^{2}+\beta^{2}-\xi \beta\right)\left[-\alpha \phi X-\beta \phi^{2} X\right]
$$

From (3.7) and (3.8), we find

$$
\left(\alpha^{2}+\beta^{2}-\xi \beta\right)\left[-\alpha \phi X-\beta \phi^{2} X\right]=0
$$

That is

$$
\left(\alpha^{2}+\beta^{2}-\xi \beta\right) \nabla_{X} \xi=0
$$

From which we state the following theorem.

Theorem3.3. In a generalized recurrent Lorentzian Trans-Sasakian manifold $M$ of non vanishing $\xi$-sectional curvature, the structure vector field $\xi$ is of constant length unity.

Proof. Since M is of non vanishing $\xi$-sectional curvature from (3.10) we have

$$
\nabla_{\mathrm{X}} \xi=0
$$


This proves that $\xi$ is a constant vector and further

$$
(\xi)=-1, \quad \text { implies } \mathrm{g}(\xi, \xi)=-1, \quad \text { that is }|\xi|=-1
$$

From which it follows that th e structure vector field $\xi$ is of unit length and this proves the theorem.

Corollary3.3. In a generalized recurrent Lorentzian $\alpha$-Sasakian manifold $M$ with $\alpha$ non zero, the structure vector field $\xi$ is of constant length unity.

Proof. From theorem 3.3 and (3.9), as $\beta=0$, it follows that

$$
-\alpha^{3} \phi \mathrm{X}=0
$$

Since $\alpha$ is non zero, $\phi X=0$ so that from (2.5) equation (3.11) follows. With similar argument as in the Theorem 3.3 , the proof follows.

Corollary3.4. In a generalized recurrent Lorentzian Sasakian manifold M, the structure vector field $\xi$ is of constant length unity.

Proof. For a Lorentzian Sasakian manifold $\alpha=1, \beta=0$ with similar arguments as in theorem 3.3 the proof follows. Corollary3.5. In a generalized recurrent Lorentzian $\beta$-Kenmotsu manifold $M$ with $\beta$ non zero, the structure vector field $\xi$ is of constant length unity.

Proof. From theorem 3.3 and (3.9), as $\alpha=0$, it follows that

$$
\beta^{3} \phi^{2} X=0
$$

Since $\beta$ is non zero, $\phi^{2} X=0$ so that from (2.5) equation (3.11) follows. With similar arguments as in the Theorem 3.3 the proof follows.

Corollary3.6. In a generalized recurrent Lorentzian Kenmotsu manifold M, the structure vector field $\xi$ is of constant length unity.

Proof. For a Lorentzian Sasakian manifold $\alpha=0, \beta=1$ with similar arguments as in theorem 3.3 the proof follows. Theorem3.4. In a generalized recurrent Lorentzian trans-Sasakian manifold (M, g) of non vanishing $\xi-$ sectional curvature, the following relation holds $\omega(\mathrm{X})\left(\alpha^{2}+\beta^{2}-\xi \beta\right)-\mu(\mathrm{X})=[2 \alpha \mathrm{X} \alpha+2 \beta \mathrm{X} \beta-\mathrm{X}(\xi \beta)]$

$$
\begin{aligned}
& -\frac{\alpha}{n}\left[(2 n-1)(\phi X) \beta-\left(\phi^{2} X\right) \alpha+(\phi X) \psi\right] \\
& \left.-\frac{\beta}{n}\left[\left\{2 n\left(\alpha^{2}+\beta^{2}\right)-\xi \beta\right\} \eta(X)+(2 n-1) X \beta-(\phi X) \alpha+2 \alpha \beta \eta(X)+X \alpha\right) \psi\right] \\
& -2 \beta \eta(X)\left(\alpha^{2}+\beta^{2}-\xi \beta\right)
\end{aligned}
$$

for any vector field $\mathrm{X} \in \chi(\mathrm{M})$.

Proof. From (3.1), we have

$$
\left(\nabla_{\mathrm{X}} \mathrm{R}\right)(\mathrm{Y}, \mathrm{Z}) \mathrm{U}=\omega(\mathrm{X}) \mathrm{R}(\mathrm{Y}, \mathrm{Z}) \mathrm{U}+\mu(\mathrm{X})[\mathrm{g}(\mathrm{Z}, \mathrm{U}) \mathrm{Y}-\mathrm{g}(\mathrm{Y}, \mathrm{U}) \mathrm{Z}],
$$

The above equation can be written as

$$
\left(\nabla_{\mathrm{X}} \mathrm{R}\right)(\mathrm{Y}, \mathrm{Z}, \mathrm{U}, \mathrm{V})=\omega(\mathrm{X}) \mathrm{R}(\mathrm{Y}, \mathrm{Z}, \mathrm{U}, \mathrm{V})+\mu(\mathrm{X})[\mathrm{g}(\mathrm{Z}, \mathrm{U}) \mathrm{g}(\mathrm{Y}, \mathrm{V})-\mathrm{g}(\mathrm{Y}, \mathrm{U}) \mathrm{g}(\mathrm{Z}, \mathrm{V})]
$$

Where $\quad \omega(X)=g(X, A), \quad \mu(X)=g(X, B)$.

Let $\left\{e_{i}: i=1,2,3 \ldots 2 n+1, e_{2 n+1}=\xi\right\}$ be the orthonormal basis of the tangent space $T_{P} M$ at any point $P$ of the manifold. Then setting $\mathrm{Y}=\mathrm{V}=\mathrm{e}_{\mathrm{i}}$ in (3.12) and taking the summation over $\mathrm{i}, 1 \leq \mathrm{i} \leq 2 \mathrm{n}+1$, we get

$$
\left(\nabla_{X} S\right)(Z, U)=\omega(X) S(Z, U)+2 n \mu(X)
$$

Putting $\mathrm{Z}=\mathrm{U}=\xi$ in $(3.13)$, we get

$$
\left(\nabla_{X} S\right)(\xi, \xi)=\omega(X) S(\xi, \xi)+2 n \mu(X)
$$

Using (2.11) in (3.14) we get

$$
\left(\nabla_{X} S\right)(\xi, \xi)=-2 n \omega(X)\left(\alpha^{2}+\beta^{2}-\xi \beta\right)+2 n \mu(X)
$$

The left hand side of (3.15) can be expanded as

$$
\left(\nabla_{X} S\right)(\xi, \xi)=\nabla_{X}(S(\xi, \xi))-S\left(\nabla_{X} \xi, \xi\right)-S\left(\xi, \nabla_{X} \xi\right)
$$

Using symmetric property of Ricci tensor $S$ (3.16) can be written as

$$
\left(\nabla_{X} S\right)(\xi, \xi)=\nabla_{X}(S(\xi, \xi))-2 S\left(\nabla_{X} \xi, \xi\right)
$$

Using (2.11) and (2.5) in (3.17) we have

$$
\begin{aligned}
\left(\nabla_{X} S\right)(\xi, \xi) & =\nabla_{X}\left[-2 n\left(\alpha^{2}+\beta^{2}-\xi \beta\right)\right]-2 S(-\alpha \phi X-\beta(X+\eta(X) \xi), \xi) \\
& =-2 n X\left(\alpha^{2}+\beta^{2}-\xi \beta\right)+2 \alpha S(\phi X, \xi)+2 \beta S(X, \xi)-2 \beta \eta(X) S(\xi, \xi)
\end{aligned}
$$

Using (2.9) and (2.11) in (3.18) we get

Substituting (3.19), in (3.15), we get

$$
\begin{aligned}
\left(\nabla_{\mathrm{X}} S\right)(\xi, \xi)= & \left.-2 \mathrm{nX}\left(\alpha^{2}+\beta^{2}-\xi \beta\right)+2 \alpha[(2 \mathrm{n}-1) \phi \mathrm{X}) \beta-\left(\phi^{2} \mathrm{X}\right) \alpha+(\phi \mathrm{X}) \psi\right] \\
& +2 \beta\left[\left\{2 \mathrm{n}\left(\alpha^{2}+\beta^{2}\right)-\xi \beta\right\} \eta(\mathrm{X})+(2 \mathrm{n}-1) \mathrm{X} \beta-(\phi \mathrm{X}) \alpha+(2 \alpha \beta \eta(\mathrm{X})+\mathrm{X} \alpha) \psi\right] \\
& +4 \mathrm{n} \beta \eta(\mathrm{X})\left(\alpha^{2}+\beta^{2}-\xi \beta\right)
\end{aligned}
$$

$$
\begin{aligned}
& 2 \mathrm{n}\left[(2 \alpha \mathrm{X} \alpha+2 \beta \mathrm{X} \beta-\mathrm{X}(\xi \beta)]-2 \alpha[(2 \mathrm{n}-1) \phi \mathrm{X}) \beta-\left(\phi^{2} \mathrm{X}\right) \alpha+(\phi \mathrm{X}) \psi\right] \\
& \quad-2 \beta\left[\left\{2 \mathrm{n}\left(\alpha^{2}+\beta^{2}\right)-\xi \beta\right\} \eta(\mathrm{X})+(2 \mathrm{n}-1) \mathrm{X} \beta-(\phi \mathrm{X}) \alpha+(2 \alpha \beta \eta(\mathrm{X})+\mathrm{X} \alpha) \psi\right] \\
& \quad-4 \mathrm{n} \beta \eta(\mathrm{X})\left(\alpha^{2}+\beta^{2}-\xi \beta\right)=2 \mathrm{n} \omega(\mathrm{X})\left(\alpha^{2}+\beta^{2}-\xi \beta\right)-2 \mathrm{n} \mu(\mathrm{X})
\end{aligned}
$$


Provided the manifold $\mathrm{M}$ is of non vanishing $\xi$-sectional curvature. Hence the proof of the theorem 3.4 follows from (3.20).

Corollary3.7. In a generalized recurrent Lorentzian trans-Sasakian manifold (M, g) of vanishing $\xi$-sectional curvature, associated vector field $\mu$ is given by

$$
\begin{aligned}
\mu(\mathrm{X})= & \frac{\alpha}{\mathrm{n}}\left[(2 \mathrm{n}-1)(\phi \mathrm{X}) \beta-\left(\phi^{2} \mathrm{X}\right) \alpha+(\phi \mathrm{X}) \psi\right] \\
& \left.+\frac{\beta}{\mathrm{n}}\left[\left\{2 \mathrm{n}\left(\alpha^{2}+\beta^{2}\right)-\xi \beta\right\} \eta(\mathrm{X})+(2 \mathrm{n}-1) \mathrm{X} \beta-(\phi \mathrm{X}) \alpha+2 \alpha \beta \eta(\mathrm{X})+\mathrm{X} \alpha\right) \psi\right]
\end{aligned}
$$

Proof. If the $\xi$-sectional curvature is zero, then from (3.20) proof follows.

Corollary3.8. There is no generalized cosymplectic manifold unless the associated 1-form $\mu$ vanishes.

Proof. Follows from Theorem 3.4.

\section{On Generalized Ricci Recurrent Lorentzian Trans-Sasakian Manifold}

In this section, we study some of the properties of generalized Ricci recurrent Lorentzian transSasakian manifold. Further some special cases of our results are studied, just to show the geometrical significance of our results.

Definition. A Riemannian manifold (M, g) is called generalized Ricci recrrent [5], if Ricci tensor S satisfies the following condition

$$
\left(\nabla_{X} S\right)(Y, Z)=\omega(X) S(Y, Z)+2 n \mu(X) g(Y, Z)
$$

where $\omega$ and $\mu$ are associated 1 -forms and these are defined by

$$
\omega(X)=g(X, A), \quad \mu(X)=g(X, B)
$$

Theorem4.1. If $M$ is a generalized Ricci recurrent Lorentzian trans-Sasakian manifold of non vanishing $\xi$ sectional curvature, then the following relation holds $\omega(X)\left(\alpha^{2}+\beta^{2}-\xi \beta\right)+\mu(X)=[2 \alpha X \alpha+2 \beta X \beta-X(\xi \beta)]$

for any vector field $\mathrm{X} \in \chi(\mathrm{M})$.

$$
\begin{aligned}
& -\frac{\alpha}{n}\left[(2 n-1)(\phi X) \beta-\left(\phi^{2} X\right) \alpha+(\phi X) \psi\right] \\
& \left.-\frac{\beta}{n}\left[\left\{2 n\left(\alpha^{2}+\beta^{2}\right)-\xi \beta\right\} \eta(X)+(2 n-1) X \beta-(\phi X) \alpha+2 \alpha \beta \eta(X)+X \alpha\right) \psi\right] \\
& -2 \beta \eta(X)\left(\alpha^{2}+\beta^{2}-\xi \beta\right)
\end{aligned}
$$

Proof. Now taking $\mathrm{Y}=\mathrm{Z}=\xi$ in (4.1), we have

Using (2.11) in (4.3) we get

$$
\left(\nabla_{X} S\right)(\xi, \xi)=\omega(X) S(\xi, \xi)+2 n \mu(X) g(\xi, \xi)
$$

$$
\left(\nabla_{X} S\right)(\xi, \xi)=-2 n \omega(X)\left(\alpha^{2}+\beta^{2}-\xi \beta\right)-2 n \mu(X)
$$

The left hand side of (4.4) can be expanded as

$$
\left(\nabla_{X} S\right)(\xi, \xi)=\nabla_{X}(S(\xi, \xi))-S\left(\nabla_{X} \xi, \xi\right)-S\left(\xi, \nabla_{X} \xi\right)
$$

Using symmetric property of Ricci tensor $S$ (4.4) can be written as

$$
\left(\nabla_{\mathrm{X}} \mathrm{S}\right)(\xi, \xi)=\nabla_{\mathrm{X}}(\mathrm{S}(\xi, \xi))-2 \mathrm{~S}\left(\nabla_{\mathrm{X}} \xi, \xi\right)
$$

Using (2.11) and (2.5) in (4.6) we have

$$
\begin{aligned}
\left(\nabla_{X} S\right)(\xi, \xi) & =\nabla_{X}\left[-2 n\left(\alpha^{2}+\beta^{2}-\xi \beta\right)\right]-2 S(-\alpha \phi X-\beta(X+\eta(X) \xi), \xi) \\
& =-2 n X\left(\alpha^{2}+\beta^{2}-\xi \beta\right)+2 \alpha S(\phi X, \xi)+2 \beta S(X, \xi)-2 \beta \eta(X) S(\xi, \xi)
\end{aligned}
$$

Using (2.9) and (2.11) in (4.7) we get

Substituting (4.8), in (4.4), we get

$$
\begin{aligned}
\left(\nabla_{\mathrm{X}} S\right)(\xi, \xi)= & \left.-2 \mathrm{nX}\left(\alpha^{2}+\beta^{2}-\xi \beta\right)+2 \alpha[(2 \mathrm{n}-1) \phi \mathrm{X}) \beta-\left(\phi^{2} \mathrm{X}\right) \alpha+(\phi \mathrm{X}) \psi\right] \\
& +2 \beta\left[\left\{2 \mathrm{n}\left(\alpha^{2}+\beta^{2}\right)-\xi \beta\right\} \eta(\mathrm{X})+(2 \mathrm{n}-1) \mathrm{X} \beta-(\phi \mathrm{X}) \alpha+(2 \alpha \beta \eta(\mathrm{X})+\mathrm{X} \alpha) \psi\right] \\
& +4 \mathrm{n} \beta \eta(\mathrm{X})\left(\alpha^{2}+\beta^{2}-\xi \beta\right)
\end{aligned}
$$

$$
\begin{aligned}
& -2 \mathrm{n}\left[(2 \alpha \mathrm{X} \alpha+2 \beta \mathrm{X} \beta-\mathrm{X}(\xi \beta)]+2 \alpha[(2 \mathrm{n}-1) \phi \mathrm{X}) \beta-\left(\phi^{2} \mathrm{X}\right) \alpha+(\phi \mathrm{X}) \psi\right] \\
& \quad+2 \beta\left[\left\{2 \mathrm{n}\left(\alpha^{2}+\beta^{2}\right)-\xi \beta\right\} \eta(\mathrm{X})+(2 \mathrm{n}-1) \mathrm{X} \beta-(\phi \mathrm{X}) \alpha+(2 \alpha \beta \eta(\mathrm{X})+\mathrm{X} \alpha) \psi\right] \\
& \quad+4 \mathrm{n} \beta \eta(\mathrm{X})\left(\alpha^{2}+\beta^{2}-\xi \beta\right)=-2 \mathrm{n} \omega(\mathrm{X})\left(\alpha^{2}+\beta^{2}-\xi \beta\right)-2 \mathrm{n} \mu(\mathrm{X})
\end{aligned}
$$

Provided the manifold $\mathrm{M}$ is of non vanishing $\xi$-sectional curvature. Hence the proof of the theorem 4.1 follows from (4.9).

Corollary4.1. A generalized Ricci recurrent Lorentzian $\beta$-Kenmotsu manifold with $\beta$ non zero constant the relation $\beta^{2} \omega+\mu=0$ holds.

Proof. Follows from Theorem 4.1.

Corollary4.2. A generalized Ricci recurrent Lorentzian $\alpha$-Sasakian manifold with $\alpha$ non zero constant the relation $\alpha^{2} \omega+\mu=0$ holds.

Proof. Follows from Theorem 4.1.

Corollary4.3. A generalized Ricci recurrent Lorentzian Kenmotsu manifold, the associated 1-forms are in the opposite direction.

Proof. From the statement of the corollary and Theorem 4.1 it follows that $\omega=-\mu$ which proves the corollary. 
Corollary4.4. A generalized Ricci recurrent Lorentzian Sasakian manifold, the associated 1-forms are in the opposite direction.

Proof. From the statement of the corollary 4.2 and Theorem 4.1 it follows that $\omega=-\mu$ which proves the corollary.

\section{References}

[1]. A. Gray and L.M. Harvella, The sixteen classes of almost Hermitian manifolds and their linear invariants,Ann.Math.Pura Appl, 123(4)(1980), 35-58.

[2]. Ahmed Yildiz, On Lorentzian $\alpha$-Sasakian manifolds,Kyungpock Math.J. 45 (2005), 95-103.

[3]. A.Y. Mine Turan and Eftal Acet A,On three dimensional Lorentzian $\alpha$ - Sasakian manifolds, Bulletin of Mathematical Analysis and Applications, ISSN 1821-1291 (2009), 90-98.

[4]. G.T. Srinivas, Venatesh and C.S. Bagewadi, On Lorentzian $\beta$ - Kenmotsu manifolds, General Mathematics, 18(4)(2010), 61-69.

[5]. Hakan Ozturk, et.al. (2010) On $\alpha$-Kenmotsu manifolds Satisfying Certain conditions pl.sciences vol. 12. , 115-126.

[6]. J.A. Oubina, New classes og almost contact metric structure, Publ. Math.Debercen, 32 (1987), 187-193.

[7]. J. A Oubina, New class of almost contact metric manifolds. Publ. Math. Debrecen, (1985), 32, 187-193.

[8]. J.C. Morrero and D. Chinea, On trans-Sasakian manifolds, Proceedings of the XIV in Spanish-Portugues Conference, Vol.-I-III (Spanish)(Puerto de la Craz, (1989), 655-659.

[9]. J.S. Kim.et.al (2002), On generalized Ricci recurrent trans-Sasakian Manifolds J.Korean Math,Soc,39(6),953.

[10]. K. Motsumoto,On Lorentzian para contact manifolds, Bull.of the Yamagata Uni,12(2)(1989), 151-156.

[11]. Q.Khan. On generalized recurrent Sasakian Manifolds, Kyungpook Math. J. 44,2(2004), 167-177.

[12]. S. Tanno, The automorphism groups of almost contact Riemannian manifolds, Tohoku Math.J.21 (1969), 21-38

[13]. S. S. Pujar, and V. J. Khairnar, On Lorentzian Trans-Sasakian manifolds-I, Ultra Scientist Vol. 23(1)M, (2011), 53-66.

[14]. S.S.Pujar and V.J.Khairnar, On Lorentzian trans-Sasakian manifold-I, Int.J.of Ultra Sciences of Physical Sciences, 23(1)(2011), 5366.

[15]. S.S. Pujar and V.J.Khairnar, On weak symmetries of Lorentzian trans-Sasakian manifolds, Acta Ciencia, 38(2) (2012), 287-300.

[16]. S.S. Pujar, On $\delta$ Lorentzian $\alpha$ Sasakian manifolds, to appear in Antactica J. of Mathematics 8(2012).

[17]. S.S.Pujar and S.S. Naik, On generalized $\phi$-recurrent, concircular $\phi$-recurrent, and Projective $\phi$-recurrent, Trans Sasakian manifolds, J. of Ultra Scientist of Physical Sciences Vol.22(3)M,(2010), 697-710.

[18]. U.C.De and N.Guha, On generalized recrrent manifolds, Proceedings of Math. Soc., 7 (1991), 7-11. 